\title{
LAS TUTORÍAS Y LA FORMACIÓN PROFESIONAL: CASO DE ESTUDIO UNIVERSIDAD TÉCNICA DE MANABÍ, PORTOVIEJO, ECUADOR.
}

\section{TUTORIALS AND PROFESSIONAL TRAINING: STUDY CASE UNIVERSIDAD TÉCNICA DE MANABÍ, PORTOVIEJO, ECUADOR.}

Kasandra Vanessa Saldarriaga Villamil, Mgs. Magíster en Administración de Empresas (Ecuador). Docente de la Universidad Técnica de Manabí, Ecuador. kasandra.saldarriaga@fci.edu.ec

María Isabel Amor Almedina, Ph.D. Doctora en Psicopedagogía (España). Docente del Departamento de Educación de la Universidad de Córdoba, España.

\section{ARTÍCULO DE INVESTIGACIÓN}

Recibido: 14 de agosto de 2018.

Aceptado: 12 de noviembre de 2018.

\section{RESUMEN}

Este trabajo pretende demostrar la conveniencia y necesidad de promover a mayor escala la tutoría en el ámbito de la Educación Superior; considerándose ésta como la estrategia que crea vínculo en los procesos de enseñanza y aprendizaje entre tutor y estudiante. La función tutorial se la caracteriza como el medio en que el docente interactúa con su tutorado con el fin de contribuir en la orientación, acompañamiento y apoyo permanente durante la estancia de formación para garantizar el desarrollo de sus competencias y la culminación exitosa del graduado. El objetivo fundamental de este estudio, es analizar la importancia de las tutorías en la formación de los profesionales de la Universidad Técnica de Manabí. La metodología utilizada es el método deductivo con enfoque cuantitativo, apoyados en el método analítico y descriptivo, para ello se ha diseñado un cuestionario que se aplicará a los estudiantes y docentes donde se han abordado diferentes dimensiones que permiten valorar los procesos de tutorías empleados en este centro de Educación Superior. Entre los resultados esperados se evidenciará la importancia de la tutoría enmarcada en el Reglamento General de Tutorías 
que regenta esta importante gestión académica, así como también las dificultades que se presentan en su desarrollo y ejecución. Las derivaciones citadas darán las pautas para establecer propuestas de mejora en el desarrollo de la tutoría que contribuyan a garantizar la formación integral los estudiantes.

Palabras clave: tutoría, orientación, competencias, formación profesional, educación superior

\section{INTRODUCCIÓN}

El mundo actual se caracteriza por cambios y trasformaciones apresuradas, estos cambios, están presididos por una serie de factores generales, tales como la globalización, el impacto de las tecnologías de la información y comunicación (TIC), la administración del conocimiento y la necesidad de gestionar la diversidad, hacen necesario un entorno educativo significativamente diferente y obligan a la universidad a repensar su rol tradicional, enfocándose en nuevas perspectivas con misión, visión y compromiso con la comunidad científica, enfocado en formar profesionales propositivos que respondan a las exigencias de la sociedad de la información y el conocimiento, donde la tutoría universitaria pasa a convertirse en una práctica didáctica, una red de apoyo que propicia un modelo de guía, orientación y acercamiento docente-estudiante en una simbiosis que genera el espacio adecuado para el aprendizaje significativo.

Emplazados en el contexto latinoamericano, los movimientos sociales, económicos y políticos dan muestras claras que la sociedad necesita contar con ciudadanos preparados cultural e intelectualmente, para hacer frente a los desafíos del presente y del futuro; así como asumir el papel que deben jugar en el desarrollo de sus países. Las universidades, por el rol que ocupan en la sociedad, son las instituciones adjudicadas para desempeñar una función clave en el diseño e implementación de estrategias adecuadas en el arribo a esta meta. Tienen la misión, y sobre todo, la responsabilidad fundamental de usar los conocimientos, la tradición y capacidad de innovación para preparar el futuro del país.

La universidad tiene el deber de reconocer que el cometido y la labor del profesorado se encuentra en constante transformación, en línea con los cambios sociales, por ello, debe responder con adecuada capacitación a los docentes en las diversas actividades de gestión académica que tiene que asumir para brindar la pertinente formación a los estudiantes y estos puedan afrontar los desafíos que le impone la sociedad. Uno de los retos es garantizar una

ISSN: 1390-9320, Vol. 6, No. 1, febrero 2019 
estructura flexible, tendiente a fortalecer la tutoría personalizada que aborde nuevos modelos de enseñanza - aprendizaje que sobrepasen los métodos tradicionales.

\section{REVISIÓN TEÓRICA}

\subsection{La orientación y la tutoría universitaria}

En la universidad, para (Álvarez, 2008; Bausela, 2010 y Sanz Oro, 2010) desde hace tiempo, la tutoría tiene la función de guiar al alumnado de manera personalizada, para fomentar su integración, facilitar su transición a la vida activa y al mundo laboral; pero los cambios derivados de la transformación europea, además de suponer un avance para la calidad en el ámbito superior, también ha originado nuevas necesidades en la propia institución, en el profesorado y sobre todo en el alumnado.

Según Rué, en opinión del estudiante, deduce que el profesorado debe cambiar del modo magistral a un modo tutorial ya que el alumnado considera necesario desvincular las tutorías de la orientación para los exámenes y asignarles nuevos contenidos, en cuanto que es un espacio para orientar los aprendizajes y asesorar sobre las futuras prácticas (Rué, 2004, p. 182).

En este sentido, Jiménez manifiesta que se debe ser más sensible a los requerimientos del estudiante universitario en todas sus dimensiones, como persona, de forma continuada y progresiva (Jiménez, 2010). "La educación afronta en estos momentos retos importantes y uno de ellos tiene que ver con los que surgen de la sociedad de la información que plantea nuevos escenarios y oportunidades en lo social, lo económico y lo cultural" (Garzón, 2012, p. 276).

La tutoría debe convertirse en un espacio de formación y no una reseña en el horario del profesor/a (Amor, 2015). Actualmente, la necesidad de la formación se extiende más allá de la orientación y el asesoramiento de contenidos ligados a las asignaturas.

Pantoja y Campoy (2009), mencionan que la universidad actual para la sociedad de hoy, necesita un nuevo modelo de enseñanza y aprendizaje. Según estos autores, se debe crear una universidad que no sólo investigue, sino que también eduque en la vida y para la vida; enseñe en lo académico y lo vital, y además, tenga en cuenta la realidad y la problemática social; poniendo la investigación y la docencia al servicio de las necesidades del alumnado y las demandas sociales (Amor, 2016a).

ISSN: 1390-9320, Vol. 6, No. 1, febrero 2019 
En varios países, los servicios de orientación y la tutoría comienzan a potenciarse en las universidades como es el caso del desarrollo del Espacio Europeo de Educación Superior (EEES), especialmente en lo que respecta a dotar de contenidos a la acción tutorial y orientadora del profesorado como función complementaria a su tarea docente (Sanz, 2010).

Esta apuesta por la orientación y la tutoría como un cambio de paradigma universitario con el que se pretende superar un modelo instructivo, basado en la transmisión de contenidos, a favor de un modelo formativo integral, centrado en la comunicación bidireccional y en la interacción profesor/alumno (Amor, 2016b).

En el marco del Espacio Europeo de Educación Superior se ha puesto de manifiesto la necesidad de promover la convergencia entre los diferentes sistemas de educación superior para facilitar, a los titulados, la integración en un mercado laboral sin fronteras y ofrecer un marco más atractivo para los estudiantes del resto del mundo (Consejo de la Unión Europea, 2004).

Este propósito plantea una nueva realidad universitaria en la que:

a. El intercambio, la movilidad, la transparencia y la comunicación se convierten en elementos claves para la calidad en las universidades.

b. Se encuentra perfiles de alumnado muy heterogéneo, con características personales, intereses, expectativas y necesidades diferentes.

c. Se propone un cambio en el paradigma educativo, donde el estudiante se sitúa en el centro del proceso del aprendizaje y se hace visible la necesidad de metodologías orientadas al aprendizaje activo.

d. El perfil académico profesional es definido a través de competencias. Con ello se pretende que la universidad de respuesta a las nuevas exigencias profesionales (saber, saber hacer, saber estar y ser).

e. Se requiere métodos de orientación, seguimiento y evaluación como elementos fundamentales para garantizar la calidad, por lo que la orientación se muestra como un ámbito perpendicular y transversal en la formación universitaria abierta al mundo en general. 
En este contexto, la tutoría académica universitaria se proyecta como una estrategia para dar respuesta a aspectos como:

- Gran diversidad en cuanto a las características de estudiantes que acceden a la universidad (edades, nacionalidades, intereses, situaciones familiares y profesionales diferentes)

- Desconocimiento de la institución, metodología y estructura organizativa, académica y relacional por parte de los alumnos de nuevo ingreso.

- Nuevos planes de estudios y definición de los itinerarios formativos

- Nuevas exigencias del mercado laboral en competencias instrumentales, personales y sociales.

En Europa los servicios de orientación y la tutoría comienzan a potenciarse en las universidades como consecuencia del desarrollo del EEES, especialmente en lo que respecta a dotar de contenido a la acción tutorial y orientadora del profesorado como función complementaria a su tarea docente (Sanz, 2005).

Además, de las ya conocidas potencialidades de las TIC como proporcionar un ambiente de comunicación flexible, independiente de barreras de espacio y tiempo (Cabero, 1999; Salinas, et. al. 2004; Gisbert, 2000); Bierema y Merriam (2002) señalan dos aspectos claves que marcan una diferencia clara entre la tutoría on-line y la tutoría tradicional cara a cara:

- Afrontamiento de nuevos retos

- Igualdad en el intercambio.

El e-learning no es una tecnología más, ya que todos los indicios apuntan a que transformará la enseñanza y el aprendizaje (Garrison y Anderson, 2005). La incorporación de las TIC en la educación supone un cambio cultural, por lo que plantean un enfoque y un modo de trabajar distintos a los tradicionales (Rodríguez Moreno, 2002), lo que posibilita y facilita la autoformación (Ardizzone y Rivotella, 2003), por tanto, las estrategias, en el ámbito tecnológico y las competencias que se desarrollan, se dirigen a alcanzar estándares internacionales.

Este sistema permite poco a poco configurar una mente virtual sustancialmente distinta a la mente letrada que conocemos, con la que interpretamos y respondemos al mundo (Monereo, 2008). 
Concibiendo el objetivo de la orientación como el de propiciar el desarrollo de las competencias necesarias para identificar, elegir y reconducir alternativas personales, académicas y profesionales, de acuerdo con el potencial y proyecto vital de cada una de las personas, contrastadas con las ofertas de los entornos formativos y socio labórales (Echeverría, 2004).

La e-tutoría debería de tener como finalidad "facilitar a los alumnos todas las herramientas y ayuda necesaria para que puedan conseguir con éxito tanto las metas académicas como personales y profesionales que les plantea la universidad" (Montserrat; Gisbert; Rallo, 2005).

La adaptación de la Universidad española al Espacio Europeo de Educación Superior (EEES), supone el abordaje de varios cambios en los procesos de enseñanza y aprendizaje, dirigidos a facilitar la autonomía, el desarrollo de competencias y la formación integral del estudiante. Estas transformaciones que se están desenvolviendo en el terreno europeo, están progresivamente alcanzado al contexto latinoamericano, como pone de manifiesto el "Proyecto Tuning América Latina" (Montaño, 2013).

Hemos evolucionado desde situaciones en las que primaban los procesos de enseñanza hasta las actuales, en las que la importancia se centra en los procesos de aprendizaje. En este escenario el rol, tanto del profesor como del alumno, está cambiando en todos los contextos educativos, el alumno se configura como pieza clave en todo su programa de formación, ya que pues su finalidad fundamental es que sea él quien alcance los objetivos propuestos (Mir, Reparaz y Sobrino, 2003). Y el profesor pasa de ser transmisor de conocimientos a orientador y guía en el aprendizaje (Amor, 2015).

Romero y LLorente (2006) y Barroso (2008), resumen las competencias y habilidades que debe tener el tutor virtual en: Competencias pedagógicas, competencias técnicas y organizativas.

Los tutores virtuales necesitarán tener competencias y habilidades para dar respuesta a las necesidades a las diferentes tareas que se le irán presentando progresivamente a lo largo de su rol como profesor-tutor (Lleixà y Gilbert, 2010).

En este marco general, la tutoría se convierte en una parte sustancial del proceso de enseñanza-aprendizaje universitario y una estrategia para promover su mejora. 
Por consiguiente, apostamos por implantar un proceso de tutoría académica que potencie una formación integral y de calidad, facilitando un modelo de aprendizaje centrado en el alumnado, donde las Tecnologías de la Información y la Comunicación (TIC) desempeñan un papel relevante.

La Tutoría Académica se entiende como un proceso de apoyo permanente que da respuesta a los problemas y necesidades relacionadas con la vida universitaria del estudiante, sobre todo en la fase de toma de decisiones. El modelo de tutoría al que hacemos referencia, debe ser un modelo flexible y accesible, tanto para el alumnado como para el profesorado y, sobre todo, debe permitir un cambio en su conceptualización y percepción por parte de la propia comunidad universitaria. Para que la tutoría cumpla el cometido para el cual fue creada, está sustentada en un PAT (plan de acción tutorial), estructurado en tres etapas: de inicio, durante $\mathrm{y}$ al final de los periodos de estancia universitaria.

Cano (2009), respecto a los tres estadios o periodos de relación del estudiante con la Universidad que constituyen, por tanto, los tres momentos esenciales de la orientación y la tutoría, destacando veinte objetivos fundamentales para el conjunto de las tres fases. Después desarrollaremos las acciones concretas para conseguir dichos objetivos.

\subsection{La tutoría en la Universidad Técnica de Manabí}

Actualmente, en el contexto iberoamericano, las instituciones universitarias se exponen a una serie de factores que hacen necesario establecer procesos y estructuras de reformas (Bianculli y Marchal, 2013) como son: el aumento en el número de alumnos/as que acceden a la universidad, sobre todo a las públicas, el incremento en la diversidad del alumnado como consecuencia de las nuevas situaciones sociales, la alta incidencia de abandono, el cambio del rol del estudiante como principal protagonista de su proceso de aprendizaje, la nueva estructuración en los planes de estudios, el nuevo rol del profesorado, los cambios en las formas de aprender del alumnado y las actuales exigencias sociales.

Todas estas transformaciones demandan de una tutoría que actúe como eje vertebrador entre la función docente y la formación integral del alumnado. Para dar respuesta a estas necesidades el rol del profesorado queda definido como tutor y asesor que asume unas responsabilidades que van más allá de la práctica docente (Árias et al., 2005). 
Ante esta situación, este trabajo pretende indagar sobre las necesidades que tiene el alumnado en la universidad y que proyecta a través de las tutorías. Es preciso, conocer las acciones que más se realizan, la demanda de los estudiantes, información que contribuirá a develar las necesidades reales para su formación y a determinar la función que cumple la tutoría en este espacio.

La importancia que la tutoría está adquiriendo en las universidades iberoamericanas se debe al auge que presenta en el contexto educativo moderno como un instrumento fundamental para proporcionar atención personalizada al estudiante y orientarle en su desarrollo personal, académico y profesional; por este motivo, entre los objetivos primordiales de la mayoría de las universidades (Michavila, 2012; Caldera Montes, Carranza Alcántar, Jiménez Padilla y Pérez Pulido, 2015), figura la acción de desarrollar estrategias de asesoramiento para el alumnado, tomando como referencia las mejores prácticas de las universidades del mundo.

Según el Reglamento General de Tutorías de la Universidad Técnica de Manabí (2015, p.1):

La tutoría es una de las funciones propias de todas las personas que ejercen la actividad docente y está caracterizada por orientar y apoyar el desarrollo integral de los/as estudiantes, así como contribuir a solucionar los problemas de reprobación, deserción y rezago.

Y establece las siguientes funciones del tutor/a independientemente de su dedicación, o tipo de profesor/a, titular o contratado:

- Brindar orientación educativa a los/as estudiantes.

- Promover sus capacidades.

- Apoyar su toma de decisiones académicas.

- Planear junto a él o ella su trayectoria académica.

- Identificar sus necesidades y poder atenderlas o derivarlas al profesional especializado.

- Establecer, junto con el responsable del Plan Institucional de Tutorías, las estrategias de apoyo para el alumnado que presente problemas de reprobación y rezago. 


\section{MATERIALES Y MÉTODOS}

De la importancia y pertinencia de profundizar en esta temática en el momento actual y del interés personal que suscita surge el presente trabajo de investigación. Su finalidad consiste en poner de manifiesto la importancia de la orientación y la acción tutorial como uno de los componentes que, con mayor eficacia, puede y debe contribuir a la formación integral del alumnado universitario (Amor, 2016c), además de responder a los desafíos y cambios que exige el nuevo escenario de Educación Superior. Por otro lado, pretende analizar el desarrollo de la tutoría en la orientación y asesoramiento del alumnado y las funciones que cumplen para satisfacer sus necesidades en la formación profesional en la Universidad Técnica de Manabí.

La tutoría y la orientación, en este sentido contribuyen a lograr estos planteamientos, originados por la convergencia europea. Por ello, se piensa que puede ser de interés analizar esta estrategia educativa, para mejorar la calidad en la formación de los estudiantes, en la medida que constituye una herramienta de apoyo al proceso de enseñanza y aprendizaje.

En criterio de López (2017) las tutorías han constituido un elemento sustantivo que da sentido a la finalidad de la universidad, al tratar de apoyar y asesorar al conjunto del estudiantado en el proceso de ajuste, adaptación, desarrollo y formación. En este sentido se puede definir a la tutoría como especie de educación personalizada que tiene como objetivo primordial constituirse en un modelo de soporte, que brinde acompañamiento al alumno para que sienta confianza, respaldo y apoyo.

La modalidad de tutoría universitaria, podría traducirse en un modelo de educación personalizada, considerando que a los alumnos se les asigna un docente-tutor, que es quien estará a cargo de su evolución académica, esta cercanía es considerada como un respaldo desde la perspectiva de los alumnos, debido a que se convierte en un elemento que promueve y afianza la seguridad de tener un guía que estará predispuesto a solventar y dilucidar dudas, o dificultades, tanto en el plano académico como personal.

En el plano académico resulta de gran beneficio el respaldo del docente- tutor, para promover un aprendizaje significativo con orientación respecto a técnicas de estudio, actividades de lectura comprensiva, estrategias de metacognición.

El docente - tutor también deberá en este modelo de enseñanza aplicar una metodología de observación para determinar el tipo de competencia más desarrollada por el alumno asignado

ISSN: 1390-9320, Vol. 6, No. 1, febrero 2019 
para su tutoría y así fomentar el aprendizaje desde esta destreza a fin de lograr mejores resultados.

Resulta imperativo realizar estudios en este campo, debido a que las condiciones y circunstancias sociales cambiantes, la utilización de las tecnologías de información y comunicación, provocan en ocasiones un distanciamiento de las personas, las tutorías vendrían a ser como un reencuentro entre docentes - alumnos, el primero como guía, mentor, orientador y el alumno el discípulo que ve en su tutor un respaldo en su camino hacia su formación profesional.

\subsection{Objetivos}

Analizar el desarrollo de la tutoría en la orientación y asesoramiento del alumnado por parte del profesorado de la UTM de Portoviejo, Manabí.

\subsubsection{Objetivos específicos:}

a) Conocer la percepción de profesorado y alumnado en cuanto el desarrollo de las tutorías.

b) Analizar las funciones que desempeña el docente a través de las tutorías.

c) Examinar los objetivos que se plantean mediante la tutela.

d) Valorar las demandas o necesidades del alumnado en esta tarea.

\subsection{Método}

\subsubsection{Población}

Para la estimación inicial de una muestra representativa se tuvo en cuenta el número total de profesorado ( $\mathrm{N}=900)$ y de estudiantes ( $\mathrm{N}=13.744)$, de la Universidad Técnica de Manabí. La muestra estará compuesta por un total de 300 profesores y 3700 estudiantes. Se ha asumido un error muestral del $5 \%$ para un nivel de confianza del $95 \%$.

\subsubsection{Instrumento}

Para la recogida de información se elaboró un cuestionario con 26 ítems y agrupados atendiendo a dos dimensiones: $1^{\text {a }}$ la tutoría como herramienta fundamental de orientación y asesoramiento al alumnado (Objetivos, funciones y dimensiones) y $2^{\underline{a}}$ las necesidades del alumnado universitario planteadas a través de las tutorías. 
En este caso, las preguntas que se plantean permiten expresar el valor de acuerdo o desacuerdo con las afirmaciones expuestas, basándose en una escala tipo Likert con diferentes categorías (1-5). Para ello, se presentan 5 alternativas de respuesta de elección única, en la que se establece una graduación de total desacuerdo (1) a totalmente de acuerdo (5).

En relación a la validez del cuestionario, se definió la eficacia del contenido a través de la técnica de expertos, como el grado en que una prueba representa de forma adecuada lo que se ha realizado. Para el análisis de fiabilidad del instrumento se obtuvo el coeficiente de alfha de Cronbach.

\subsection{Procedimiento}

La aplicación del cuestionario se realizará de forma presencial en horario lectivo en clase para los estudiantes, y con cita previa para el profesorado. El anonimato de ambos participantes estará garantizado en todo el proceso y esta tarea será desempeñada por la investigadora.

En cuanto a los análisis estadísticos realizados para alcanzar el objetivo de este estudio, el procedimiento será el siguiente: en primer lugar, se realizará el AFC (Análisis Factorial Confirmatorio) de la escala que evalúa el desarrollo de la tutoría en la UTM. En segundo lugar y para someter a contraste la estructura factorial del instrumento utilizado, se realizarán análisis estadísticos descriptivos básicos y se calculará la fiabilidad de la escala (alfa de Cronbach).

Para ello, serán tenidas en cuenta las recomendaciones de Hu y Bentler (1999), en las cuales un modelo se ajusta adecuadamente a los datos observados cuando el estadístico chicuadrado en comparación con sus grados de libertad presenta una ratio inferior a cinco, así como RMSEA (Root Mean Square Error of Approximation), cuyo valor recomendable es <.08, los índices NNFI (Non-NormedFit Index), IFI (Incremental Fit Index) y CFI (Comparative Fit Index), cuyos valores recomendables son >.95 (Jöreskog y Sörbom, 1993).

En tercer lugar, como método de estimación se utilizará el de máxima verosimilitud robusta. Y, posteriormente se realizará la comparación de medias en función de las variables: sexo, edad, carrera y experiencia docente del profesorado, así como, sexo y carrera en el alumnado.

Los programas informáticos utilizados en el mencionado proceso serán: el SPSS 18 para los análisis de fiabilidad, descriptivos y comparativos, y la versión 6.1 del programa estadístico 
EQS para los análisis factoriales confirmatorios.

\subsection{Cronograma/Planificación}

A continuación, en la figura 1 se puede observar las diferentes fases en las que se llevará a cabo la planificación del Proyecto de Investigación:

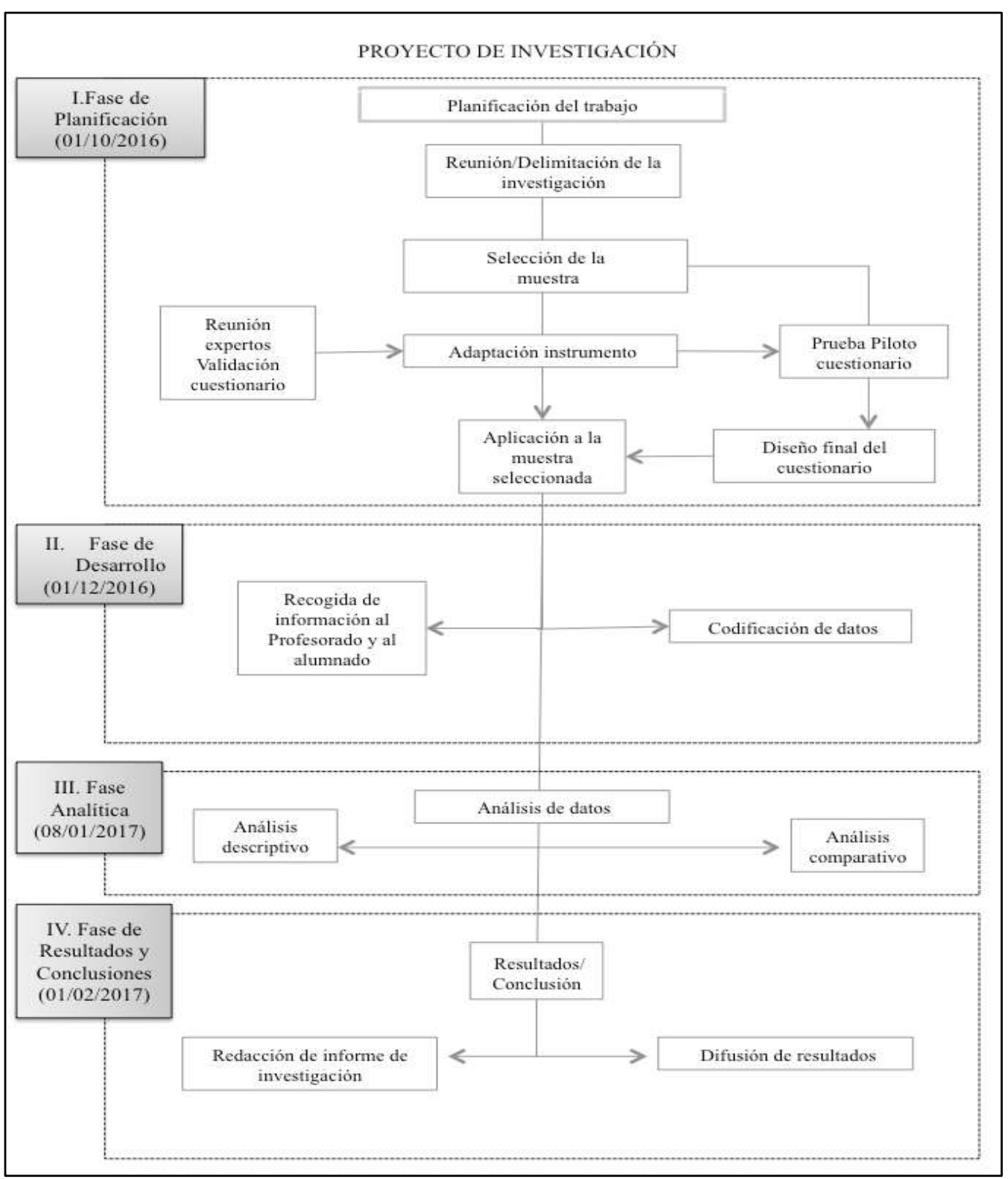

Figura 1. Fases del Proyecto de Investigación en la UTM 


\section{RESULTADOS ESPERADOS}

Con este trabajo de investigación se pretende conseguir los objetivos planteados en su inicio. En primer lugar, conocer el desarrollo de la tutoría en la Universidad Técnica de Manabí (UTM) $y$, en segundo lugar, aproximarnos a los objetivos que se cumplen y a las funciones que desempeña el profesorado a través de ellas.

Con los resultados obtenidos también se espera lograr un cambio en la cultura universitaria de la UTM a través de la tutoría como espacio para la formación integral del alumnado, es decir, concebir la tutoría como factor preponderante en la calidad de formación que se brinda en la institución.

A su vez, se pretende potenciar el desarrollo profesional de su profesorado y plantear propuestas de formación para el desarrollo de competencias relacionadas con la orientación y el asesoramiento al alumnado. Según el Reglamento general de Tutorías de la Universidad Técnica de Manabí (2015, p. 3) el perfil del docente tutor/a debe tener las siguientes características:

1. Empoderamiento con la actividad tutorial, estar comprometido con los fines de la institución.

2. Capacidad para manejar asertivamente los conflictos, inquietudes y sugerencias de los estudiantes.

3. Ser creativo para mantener el interés del estudiante.

4. Reconocer la participación y el esfuerzo de éstos.

5. Ser capaz de crear un ambiente de confianza y respeto con los estudiantes.

6. Poseer empatía.

7. Tener capacidad de liderazgo.

Por último y más importante, se espera contribuir al mejoramiento de los resultados académicos y la optimización del rendimiento de los educandos UTM.

Con estos resultados, este trabajo determinará que la tutoría académica puede constituirse en un elemento importante en el desarrollo de las actividades académicas del docente, ya sea dentro del aula, como en ambientes de aprendizaje diseñados en los ámbitos laborales y académicos. Con la finalidad de contribuir a la eficiencia y eficacia en los procesos de enseñanza aprendizaje, fortaleciendo las capacidades para la organización y la integración 
del conocimiento teórico y profesional en el alumnado. Por lo tanto, la tutoría se convertirá en un factor o mecanismo que permitirá mejorar el proceso de enseñanza y aprendizaje. Las tutorías son conceptualizadas desde diferentes formas, sin embargo, todas ellas se relacionan con la orientación, la colaboración, el acompañamiento y la asesoría académica, de forma grupal e individual.

\section{DISCUSIÓN Y CONCLUSIÓN}

La panorámica de las universidades en los países que constituyen el espacio iberoamericano no es monocolor. Por eso, de los diversos informes e investigaciones realizados en los últimos años, se perfilan unos logros y unos ritmos desiguales según países, contextos y universidades (Lobato y Guerra, 2016 y Amor, y Dios, 2017).). Como en estudios anteriores (González Tirados y González Maura, 2007), este estudio permitirá identificar las principales necesidades de formación docente del profesorado para acometer los retos planteados por el EEES, las cuales se concentran en: insuficiencias en el dominio de la acción tutorial, en el conocimiento de la personalidad del estudiante y de los procesos de liderazgo, trabajo en equipo y comunicación, en la utilización de las TIC como apoyo en el proceso de aprendizaje y de las tutorías, así como en el manejo de técnicas para la innovación educativa.

Numerosos estudios realizados en el contexto universitario latinoamericano (Soto, 2009 y Moscoso; Hernández, 2015 y Amer, 2017), han demostrado que la preocupación institucional en el ejercicio profesional del docente universitario se orienta fundamentalmente al hecho de dictar la cátedra. De esta manera, encontramos un promedio de 16 horas en los profesores de planta y de 24 en los profesores contratados. Es decir, al docente se le establece como actividad prioritaria el hecho de "impartir clases".

Cada vez está más clara la importancia de la formación pedagógica del docente universitario, sin embargo, según el estudio realizado por Moscoso y Hernández (2015) en las instituciones universitarias de Ecuador, dicha formación ha perdido relevancia, ya que se privilegia la disciplinar.

Se requiere elaborar una plan de acción tutorial, porque este permite lograr beneficios bidimensionales entre el estudiante, el profesor tutor y la institución que se beneficia de la relación tutorial establecida entre tutor el estudiante y la institución, genera métodos de acompañamiento colaborativos basados en trayectorias estudiantiles, profesionales y el sistema educativo por la que se obtiene beneficios académicos que pueden ser plasmados

ISSN: 1390-9320, Vol. 6, No. 1, febrero 2019 
en la reducción de las tasas de deserción, repitencia, favorece los procesos de investigación mejora la calidad de la educación y formación del profesional.

\section{REFERENCIAS BIBLIOGRÁFICA}

Álvarez, M. (2008). La tutoría académica en el Espacio Europeo de Educación Superior. Revista Interuniversitaria de Formación del Profesorado, 22 (1), 77-88.

Amer, J. (2017). Los procesos de implementación de los programas de mejora del aprendizaje y el rendimiento (PMAR). Perspectivas del profesorado, el alumnado y las familias. Revista Española de Orientación y Psicopedagogía (REOP). 28(3),133 - 150.

Amor, M.I. y Dios, I. (2017). La tutoría universitaria: un espacio para la orientación personal, académica y profesional en la formación inicial del profesorado. Revista Española de Orientación y Psicopedagogía, 28(1), 119-130.

Amor, M.I. (2016a) Evaluación de la orientación y la tutoría en la Facultad de Ciencias de la Educación de la Universidad de Córdoba. Educativo Siglo XXI, 34, 93-112.

Amor, M.I. (2016b). La competencia tutorial como parte de la función docente en la Educación Superior. En R. Serrano, E. Gómez y C. Huertas. La educación sí importa en el siglo XXI. (103-112). Madrid. Síntesis.

Amor, M.I. (2016c). La Tutoría universitaria. Modelo de Orientación Integral. En R. Serrano, E. Gómez y C. Huertas. La educación sí importa en el siglo XXI. (183-190). Madrid. Síntesis.

Amor, M.I. (2015). La Orientación y la Tutoría Universitaria como elementos para la calidad y la innovación en la Educación Superior. Modelo de Acción Tutorial. Teoría de la Educación, 27, 247-253.

Ardizzone, P. y Rivoltella, P. (2003). Didáctica para e-learning. Métodos e instrumentos para la innovación universitaria. España: Aljibe.

Árias, M.; Álvarez, P.R; García, M.; Cabrera, J.; Martín, M.D.; y Robayna, M. (2005). La tutoría como respuesta a las necesidades del alumnado universitario: un estudio en el primer curso del Grado de Enfermería en la Universidad de la Laguna. Revista Española de Orientación y Psicopedagogía (REOP), 16 (2), 319-331. 
Bausela, E. (2010). Planes de Acción Tutorial en la Universidad. Revista de la Educación Superior, 39 (153), 119-122.

Bianculli, K. y Marchal, M. (2013). Las tutorías universitarias: estudio de caso: Programa de Tutorías PACENI de la Facultad de Ciencias Económicas y Sociales de la UNMdP. Mar del Plata: Universidad Nacional de Mar del Plata.

Bierema, L. L., Merriam, S. B. (2002). Virtual mentoring: Using technology to enhance the mentoring process. Innovative Higher Education, 26(3), 211-227.

Cabero, J. (1999). Tecnología educativa. Madrid: Síntesis.

Caldera, J. F.; Carranza Alcántar, M. R.; Jiménez Padilla, A. A. y Pérez Pulido, I. (2015). Actitudes de los estudiantes universitarios ante la tutoría: Diseño de una escala de medición. Revista de la Educación Superior, 44 (173), 103-124.

Consejo de la Unión Europea. (2004). Proyecto de Resolución del Consejo y de los Representantes de los Estados miembros reunidos en el seno del Consejo, sobre el fortalecimiento de las políticas, sistemas y prácticas en materia de orientación permanente en Europa. http://ec.europa.eu/education/policies/2010/doc/resolution2004 es.pdf

Garrison, D. R. y Anderson, T. (2005). El E-learning en el siglo XXI. Investigación y práctica. Barcelona: Octaedro.

Garzón, R. (2012). Alfabetización digital del profesor universitario mexicano. Apuntes iniciales. Revista de Pedagogía, 33 (94), 273-288.

Gisbert, M. (2000). El profesor del siglo XXI: de transmisor de contenidos a guía del ciberespacio. En Cabero, J. (coord.). Las Nuevas Tecnologías para la mejora educativa. Sevilla: Kronos.

González Tirados, R. y González Maura, V. (2007) Diagnóstico de necesidades y estrategias de formación docente en las universidades. Revista Iberoamericana de Educación, 43 (6), 1-14.

Hu, L., \& Bentler, P. M. (1999). Cutoff criteria for fit indexes in covariance structure analysis: Conventional criteria versus new alternatives. Structural Equation Modeling. $A$ Multidisciplinary Journal, 6, 1-55. 
Jiménez, J. (2010). Hacia un nuevo Proyecto de Tutoría Universitaria en el EEES. Revista Española de Orientación y Psicopedagogía (REOP), 21(1), 37-44.

Jöreskog, K. G., \& Sörbom, D. (1993). LISREL 8: Structural equation modeling with the SIMPLIS command language. Hillsdale, NJ: Lawrence Erlbaum Associates Publishers.

Lobato, C. y Guerra, N. (2016) La tutoría en la educación superior en Iberoamérica: Avances y desafíos. Educar, 52 (2), 379-398.

López-Gómez E. (2017) El concepto y las finalidades de la tutoría universitaria: una consultaa expertos. Revista Española de Orientación y Psicopedagogía (REOP), 28(2),61-78.

Martínez, P.; Pérez, J. y González, N. (2019). ¿Qué necesita el alumnado de la tutoría universitaria? Validación de un instrumento de medida a través de un análisis

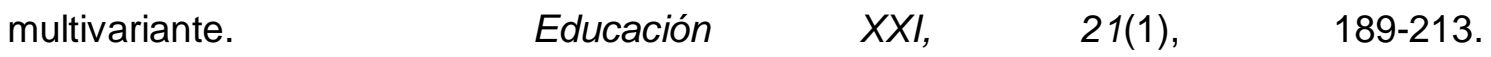
DOI: $\underline{\text { https://doi.org/10.5944/educxx1.21302 }}$

Michavila, F. (2012). Análisis de las políticas y estrategias de acogida e integración de los estudiantes de nuevo ingreso en las universidades españolas (informe EA2011- 0072). Madrid: Ministerio de Educación, Cultura y Deporte.

Monereo, C. (eds.) (2008). Psicología de la Educación Virtual. Madrid. Morata.

Montserrat, S., Gisbert, M., Cester, J. y Rallo, R. e-Tutor (2005). Towards a Tool to facilitate the Development of Academic Support Processes at the University Level. m-ICTE'05 3rd International Conference on Multimedia and ICTs in Education. Caceres, June 2005, Spain.

Moscoso, F. y Hernández, A. (2015) La formación pedagógica del docente universitario: Un reto del mundo contemporáneo. Revista Cubana de Educación Superior, 3, 140-154.

Pantoja, A. y Campoy, T. (2009) Planes de Acción Tutorial en la universidad Pantoja. Universidad de Jaén: Servicio de Publicaciones e Intercambio.

Reglamento General de Tutorías de la Universidad Técnica de Manabí. (2015, actualizado). 
Rodríguez, M.L. (2002). Hacia una nueva orientación universitaria. Modelos integrados de orientación curricular y construcción del proyecto profesional. Barcelona: Edicions Universitat de Barcelona.

Rué, J. (2004) Conceptualizar el aprendizaje y la docencia en la Universidad mediante los ETCS. Revista Interuniversitaria de Formación del Profesorado, 18 (3), 179-195.

Salinas, J.; Aguaded, J. I. y Cabero, J. (coords.) (2004). Tecnologías para la educación. Diseño, producción y evaluación de medios para la formación docente. Madrid: Alianza Editorial

Sanz, R. (2010). El profesor como tutor: un reto a consolidar en el ejercicio profesional de la orientación. Revista Española de Orientación y Psicopedagogía (REOP), 21(2), 346357. Sanz, J. (2005). Nuevas Tecnologías Aplicadas al Asesoramiento Vocacional. Diseño de Software y Servicios de Apoyo Telemático. Tesis doctoral. Universitat Jaume I de Castellón.

Soto, D. (2009) El profesor universitario de América Latina. Rhela, 13, 166-188. 